\title{
Higher-curvature corrections to holographic entanglement with momentum dissipation
}

\author{
M. Reza Tanhayi ${ }^{1,2, a}$, R. Vazirian ${ }^{1}$ \\ ${ }^{1}$ Department of Physics, Faculty of Basic Science, Islamic Azad University Central Tehran Branch (IAUCTB), P.O. Box 14676-86831, Tehran, \\ Iran \\ ${ }^{2}$ School of Physics, Institute for Research in Fundamental Sciences (IPM), P.O. Box 19395-5531, Tehran, Iran
}

Received: 22 March 2017 / Accepted: 6 February 2018 / Published online: 23 February 2018

(C) The Author(s) 2018. This article is an open access publication

\begin{abstract}
We study the effects of Gauss-Bonnet corrections on some nonlocal probes (entanglement entropy, $n$ partite information and Wilson loop) in the holographic model with momentum relaxation. Higher-curvature terms as well as scalar fields make in fact nontrivial corrections to the coefficient of the universal term in entanglement entropy. We use holographic methods to study such corrections. Moreover, holographic calculation indicates that mutual and tripartite information undergo a transition beyond which they identically change their values. We find that the behavior of the transition curves depends on the sign of the Gauss-Bonnet coupling $\lambda$. The transition for $\lambda>0$ takes place in larger separation of subsystems than that of $\lambda<0$. Finally, we examine the behavior of modified part of the force between external point-like objects as a function of Gauss-Bonnet coupling and its sign.
\end{abstract}

\section{Contents}

1 Introduction ................... . 1

2 Entanglement entropy for black brane solutions: a short review .............. 2

3 Gauss-Bonnet gravity with linear scalar fields . . . 3

3.1 HEE of a strip . . . . . . . . . . . . . . . 4

3.1.1 Low-thermal excitation $\left(m \ell^{4} \ll 1\right)$. . . 5

3.2 HEE of a sphere . . . . . . . . . . . . . . 6

3.3 HEE of a cylinder . . . . . . . . . . . . 6

4 Holographic $n$-partite Information and Wilson loop . 7

4.1 Holographic mutual information . . . . . . . 8

4.2 Holographic tripartite information . . . . . . 8

4.3 Wilson loop ... . . . . . . . . . 10

5 Conclusion ................. 11

Appendix ................. . 12

References ................. 12

a e-mail: mtanhayi@ipm.ir

\section{Introduction}

The Anti-de Sitter (AdS)/conformal field theory (CFT) correspondence postulates a relationship between quantum physics of strongly correlated many-body systems and the classical dynamics of gravity which lives in one higher dimension [1]. Through this correspondence, a great deal of progress has been made in understanding the dynamics of strongly coupled gauge theories and it has also been further extended to cover topics related to the condensed matter theory [2-5]. Actually, understanding the phenomena of strongly coupled systems in condensed matter physics might be considered as one important goal of gauge/gravity duality. Particularly, within the holographic point of view, much attention has been paid to the description of systems with momentum relaxation. Generically, on the gravity side, the solutions of Einstein-Maxwell-dilaton theories have been frequently employed to address the states of the underlying field theory. The solutions of such theories have in fact a net amount of charge and are fully translational invariant, so that a small perturbation, such as turning on an electric field, could result in an infinite DC conductivity. It is obvious that such a model cannot present a realistic description of real physical systems. In condensed matter materials, due to impurities or a lattice structure, the momentum is not conserved, which leads to a finite DC conductivity. Thus, to give a realistic description of materials in many condensed matter systems, translational symmetry must be broken. This can be done, for example, by breaking the translational invariance property [6-13]. In this direction, Andrade and Withers presented a simple holographic model for momentum relaxation [14]. Their model consists of Einstein-Maxwell theory in $(d+1)$ dimensional bulk space together with $d-1$ massless scalar fields. The neutral scalar fields in the bulk theory are dual to some operators with spatially dependent sources $\phi\left(x^{i}\right)$. These spatial sources can be chosen in such a way that the 
bulk stress tensor and hence the resulting black brane geometry are homogeneous and isotropic. The momentum relaxation concept is realized through these spatially dependent sources. Precisely, in this case it is shown that the holographic stress tensor obeys a conservation equation with contributions from the scalar vacuum expectation value $\left\langle O_{\phi}\right\rangle$

$\nabla_{i}\left\langle T^{i j}\right\rangle=\left\langle O_{\phi}\right\rangle \nabla^{j} \phi$

which is indeed the modified Ward identity where $i, j$ label the boundary space-time directions. Noting that the Ward identity yields momentum conservation in the translationally invariant solution, its modification in this sense results in breaking the translational invariance of the theory.

Inserting scalar fields into the theory in fact leads to a deformation of states at the corresponding dual field theory and it would be a relevant question what happens to some specific concepts coming from holographic computations. For example some nonlocal measures of entanglement in such a model have recently been studied in [15] via the holographic methods.

The entanglement entropy in quantum field theories is an important quantity but difficult to compute in general. However, in strongly coupled field theories, one can use holographic methods to calculate such nonlocal quantities. For example, to compute the holographic entanglement entropy (HEE) in the Einstein theory of gravity, there is an elegant proposal made by Ryu and Takayanagi (RT) [16]. According to the RT proposal, for a definite entangling region in the boundary, the entanglement entropy is related to the minimal surface $\mathcal{A}$, in the bulk whose boundary coincides with the boundary of the entangling region,

$S=\frac{\mathcal{A}}{4 G_{N}}$

where $G_{N}$ stands for Newton's constant. ${ }^{1}$ The above formula only works for CFTs dual to Einstein gravity. In such theories, the central charges are the same, since on the gravity side there are no extra parameters to distinguish the central charges. By expanding the parameter space of the couplings one can address this problem, which can be done by introducing higher-derivative corrections in the action [1823]. Thus, to study general field theories in the context of holography, higher-derivative terms are in fact needed at the gravity side. In general, higher-derivative terms could potentially introduce ghost degrees of freedom; however, it is well known that a special combination of curvature squared terms, namely the Einstein Gauss-Bonnet theory, leads to secondorder equations of motion and the theory is free of ghosts. Holographically, the Gauss-Bonnet (GB) term plays the role

\footnotetext{
${ }^{1}$ In the extended version of RT proposal named the HRT proposal, for time-dependent geometries, one should use the extremal surface [17].
}

of leading-order corrections to the Einstein gravity and in the context of AdS/CFT, the GB background is dual to a theory with different central charges, i.e., $a$ - and $c$-functions; it is noted that AdS solutions resulting from Einstein-Hilbert action yield the same $a$ and $c$ [24]. Motivated by the fact that adding higher-curvature terms into the action may help to investigate several new aspects of the theory, in this paper, we consider certain nonlocal probes of entanglement in momentum relaxation theories when the action contains GB term. More precisely, we study the holographic entanglement entropy (HEE), mutual and tripartite information; we also make a comment on the potential between external objects by computing the expectation value of Wilson loop. We find the semianalytic expression for the coefficient of universal term in HEE which could introduce a modified ' $c$ 'type central charge in the corresponding dual quantum field theory.

In order to compute HEE in the semiclassical regime when some higher-order derivative terms are added into the Einstein gravity, RT proposal should be replaced by some other recipes [19,25-28]. Some related work on this subject can be found, for example, in [29-32] and the references therein.

In this paper, we will follow the proposal of [26] to study the HEE which will be reviewed in Sect. 2. We will focus on GB gravity theory with momentum relaxation and compute the HEE for strip, spherical and cylindrical entangling regions in Sect. 3. In Sect. 4, other measurements of quantum entanglement in this setup will be considered, i.e., mutual and tripartite information and their quantum phase transitions and also the Wilson loop. In fact, we are interested in the effect of GB corrections to these quantities in holographic theories with momentum relaxation. The subject is concluded in Sect. 5. Finally, in a short appendix we present some mathematical details.

\section{Entanglement entropy for black brane solutions: a short review}

Entanglement entropy is an important nonlocal measure of different degrees of freedom in a quantum mechanical system [33]. This quantity similar to other nonlocal quantities, e.g., Wilson loop and correlation functions, can also be used to classify the various quantum phase transitions and critical points of a given system [34].

To define entanglement entropy in its spatial (or geometric) description, let us divide a constant time slice into two spatial regions $A$ and $B$ complementing each other. Thus, the corresponding total Hilbert space can be written in a specific partitioning as $\mathcal{H}=\mathcal{H}_{A} \otimes \mathcal{H}_{B}$. By integrating out the degrees of freedom that live in the complement of $A$, the reduced density matrix for region $A$ can be computed as $\rho_{A}=\operatorname{Tr}_{B} \rho$ where $\rho$ is the total density matrix. The entanglement entropy 
is given by the Von Neumann formula for this reduced density matrix as follows:

$S=-\operatorname{Tr} \rho_{A} \log \rho_{A}$.

For local $d$-dimensional quantum field theories, the entanglement entropy follows the area law and it is infinite; the structure of the infinite terms are generally as follows $[19,35,36]$ :

$$
\begin{aligned}
S(V)= & \frac{g_{d-2}\left(\mathcal{A}_{A}\right)}{\epsilon^{d-2}}+\cdots+\frac{g_{1}\left(\mathcal{A}_{A}\right)}{\epsilon} \\
& +g_{0}\left(\mathcal{A}_{A}\right) \ln \epsilon+s(V),
\end{aligned}
$$

where $\epsilon$ is the UV cutoff, $\mathcal{A}_{A}$ and $V$ stand for the area and volume of the entangling region in the boundary, $s(V)$ is the finite part of entropy and $g_{i}\left(\mathcal{A}_{A}\right)$ are local and extensive functions on the boundary of entangling region, which are homogeneous of degree $i$. The coefficient of the most divergent term is proportional to the area of the entangling surface and this is indeed the area law which is due to the infinite correlations between degrees of freedom near the boundary of the entangling surface. The coefficients of infinite terms are not physical whereas the coefficient of the logarithmic term is physical and universal in the sense that it is not affected by cutoff redefinitions.

Although computing the entanglement entropy in the context of field theory is indeed a difficult task, thanks to the AdS/CFT correspondence one can use the RT proposal to find HEE. However, as mentioned in the introduction, for actions with higher-derivative terms, one should use other proposals to compute HEE. For example, in the case of curvature squared terms with the following action:

$$
\begin{aligned}
\mathcal{I}= & \frac{1}{16 \pi G_{N}} \int_{M} d^{d+1} x \sqrt{-g}\left[R-2 \Lambda+a R^{2}\right. \\
& \left.+b R_{\mu \nu} R^{\mu \nu}+c R_{\mu \nu \rho \sigma} R^{\mu \nu \rho \sigma}-\frac{1}{2} \sum_{i=1}^{d-1}\left(\partial \phi_{i}\right)^{2}\right],
\end{aligned}
$$

pursuing the proposal of [26], HEE is given by

$$
\begin{aligned}
S= & \frac{A(\Sigma)}{4 G_{N}}+\frac{1}{4 G_{N}} \int_{\Sigma} \sqrt{\sigma} d^{d-1} x[2 a R \\
& +b\left(R_{\mu \nu} n_{i}^{\mu} n_{i}^{\nu}-\frac{1}{2} \sum_{i}\left(\operatorname{Tr} \mathcal{K}^{(i)}\right)^{2}\right) \\
& \left.+2 c\left(R_{\mu \nu \alpha \beta} n_{i}^{\mu} n_{i}^{\alpha} n_{j}^{\nu} n_{j}^{\beta}-\sum_{i} \mathcal{K}_{\mu \nu}^{(i)} \mathcal{K}_{(i)}^{\mu \nu}\right)\right] .
\end{aligned}
$$

In the above equations the cosmological constant is $\Lambda=$ $-\frac{d(d-1)}{2 L}, \phi_{i}$ are the minimally coupled massless scalar fields, $\sigma$ is the induced metric determinant, $n_{i}(i=1,2)$ are the orthogonal normal vectors on the codimension two hypersurface $\Sigma$ and $\mathcal{K}_{\mu \nu}^{(i)}$ are the extrinsic curvature tensors on $\Sigma$ defined as
$\mathcal{K}_{\mu \nu}^{(i)}=h_{\mu}^{\lambda} h_{\nu}^{\rho}\left(n_{i}\right)_{\lambda ; \rho}, \quad h_{\mu}^{\lambda}=\delta_{\mu}^{\lambda}+\xi \sum_{i}\left(n_{i}\right)_{\mu}\left(n_{i}\right)^{\lambda}$,

where $\xi$ is +1 for time-like and -1 for space-like vectors. It is noted that the first term in (2.4) is just the RT formula.

The corresponding equations of motion of (2.3) are given by

$$
\begin{aligned}
& \nabla_{\alpha} \nabla^{\alpha} \phi_{i}=0, \\
& R_{\mu \nu}-\frac{1}{2} g_{\mu \nu} R+\Lambda g_{\mu \nu} \\
& \quad-\frac{1}{2} g_{\mu \nu}\left(a R^{2}+b R_{\alpha \beta} R^{\alpha \beta}+c R_{\alpha \beta \gamma \sigma} R^{\alpha \beta \gamma \sigma}\right) \\
& \quad+2 a R_{\mu \nu} R-4 c R_{\mu}^{\alpha} R_{\nu \alpha} \\
& \quad+(2 b+4 c) R^{\alpha \beta} R_{\mu \alpha \nu \beta}+2 c R_{\mu}^{\alpha \beta \gamma} R_{\nu \alpha \beta \gamma} \\
&+\left(2 a+\frac{b}{2}\right) g_{\mu \nu} \nabla_{\alpha} \nabla^{\alpha} R+(b+4 c) \nabla_{\alpha} \nabla^{\alpha} R_{\mu \nu} \\
&-(2 a+b+2 c) \nabla_{\nu} \nabla_{\mu} R \\
& \quad+\sum_{i=1}^{d-1}\left(\frac{1}{4} g_{\mu \nu} \partial_{\alpha} \phi_{i} \partial^{\alpha} \phi_{i}-\frac{1}{2} \partial_{\mu} \phi_{i} \partial_{\nu} \phi_{i}\right)=0 .
\end{aligned}
$$

It is worth mentioning that the contribution of scalar fields to the stress tensor is supposed to be homogeneous, thus one gets a homogeneous and isotropic black brane solution. The solution can be written as

$d s^{2}=\frac{L^{2}}{\rho^{2}}\left(-f(\rho) d t^{2}+\frac{1}{f(\rho)} d \rho^{2}+\sum_{i=1}^{d-1} d x_{i}{ }^{2}\right)$,

where $f(\rho)$ is a certain function of $\rho$ and we will return to this solution later.

Since we are specifically interested in studying the GB corrections in holographic theories with momentum relaxation, in what follows, we will limit ourselves to the fivedimensional GB gravity in the bulk with three specific scalar fields which are responsible for breaking the translational invariance in the dual field theory.

\section{Gauss-Bonnet gravity with linear scalar fields}

The GB gravity can indeed be obtained by setting $a=c=$ $-\frac{b}{4} \equiv \frac{\lambda}{2} L^{2}$ in (2.3), where $\lambda$ is a dimensionless coupling constant that controls the strength of the GB term. The fivedimensional GB gravity is the simplest example of a Lovelock action and it is itself important because, in a given background, the equations of motion for a propagating perturbation contain only two derivatives.

We work with the following Einstein GB scalar gravitational action: 


$$
\begin{aligned}
\mathcal{I}= & \frac{1}{16 \pi G_{N}} \int_{M} d^{5} x \sqrt{-g}\left[R+\frac{12}{L^{2}} .\right. \\
& +\frac{\lambda L^{2}}{2}\left(R_{\mu \nu \rho \sigma} R^{\mu \nu \rho \sigma}-4 R_{\mu \nu} R^{\mu \nu}+R^{2}\right) \\
& \left.-\frac{1}{2} \sum_{i=1}^{3}\left(\partial \phi_{i}\right)^{2}\right]
\end{aligned}
$$

where the action contains massless scalar fields to incorporate momentum relaxation in the system and they are considered to be linearly dependent on spatial coordinates, i.e.,

$\phi_{i}=a_{i} x_{1}+b_{i} x_{2}+c_{i} x_{3}$.

Such an ansatz for massless scalar sources guarantees the solution to be homogeneous and isotropic. According to AdS/CFT dictionary, massless scalar fields are dual to marginal operators of the corresponding field theory and it was argued in [14] that such a spatially dependent scalar field in the bulk modifies the Ward identity, which leads to breaking of the translational invariance in the dual field theory.

The relevant equations of motion for (3.1) can simply be obtained from (2.6) and the theory admits an asymptotically $\mathrm{AdS}_{5}$ black brane solution as (2.7) in which $f(\rho)$ is given by

$f(\rho)=\frac{1-\sqrt{1-4 \lambda g(\rho)}}{2 \lambda}$,

where

$g(\rho)=1-\frac{\alpha^{2} \rho^{2}}{4}-m \rho^{4}, \quad m=\frac{1}{\rho_{h}{ }^{4}}\left(1-\frac{\alpha^{2} \rho_{h}{ }^{2}}{4}\right)$,

with $\rho_{h}$ being the radius of the horizon and the constants $a_{i}, b_{i}$ and $c_{i}$ satisfy the following relations:

$$
\begin{aligned}
& \sum_{i=1}^{3} a_{i}^{2}=\sum_{i=1}^{3} b_{i}^{2}=\sum_{i=1}^{3} c_{i}^{2}=\alpha^{2}, \\
& \sum_{i=1}^{3} a_{i} b_{i}=\sum_{i=1}^{3} a_{i} c_{i}=\sum_{i=1}^{3} b_{i} c_{i}=0 .
\end{aligned}
$$

It is noted that $f\left(\rho_{h}\right)=0$ and the UV boundary is defined as $\rho \rightarrow 0$ and the temperature of the black brane is given by

$T=\frac{1}{\pi \rho_{h}}\left(1-\frac{\alpha^{2} \rho_{h}^{2}}{8}\right)$

There is an interesting feature for the momentum relaxation methods, i.e., at zero temperature one gets $f\left(\rho_{h}\right)=$ $\left.\frac{d}{d \rho} f(\rho)\right|_{\rho=\rho_{h}}=0$, which is an extremal black brane. Although there is no $U(1)$ charge to produce an extremal black brane solution in this case, the momentum relaxation parameter gives us such a feature similar to the case of the RN-AdS black brane.

In the model that we are considering there are two deformations in the field theory due to the momentum relaxation parameter and GB term. In the following, we will develop the behavior of HEE of a quantum field theory whose states are in fact under the excitation of both momentum relaxation and GB term.

\subsection{HEE of a strip}

In order to compute $\mathrm{HEE}$, let us consider the following strip entangling region:

$$
-\frac{\ell}{2}<x_{1} \equiv x<\frac{\ell}{2}, \quad-\frac{H}{2}<x_{2} \text { and } x_{3}<\frac{H}{2},
$$

where we assume $H \gg \ell$ and $H$ plays an infrared regulator distance along the entangling surface. The corresponding codimension two hypersurface in a constant time slice can be parametrized by $x_{1}=x(\rho)$; therefore, the induced metric becomes

$d s_{\text {ind }}^{2}=\frac{L^{2}}{\rho^{2}}\left[\left(x^{\prime 2}+f^{-1}\right) d \rho^{2}+d x_{2}^{2}+d x_{3}^{2}\right]$,

where the prime stands for the derivative with respect to $\rho$. After doing some computation which are partially given in the appendix, the entropy functional is found as follows ${ }^{2}$ :

$$
\begin{aligned}
S= & \frac{H^{2} L^{3}}{4 G_{N}} \int d \rho \frac{\sqrt{x^{\prime 2}+f^{-1}}}{\rho^{3}} \\
& \times\left(1-2 \lambda \frac{f\left(f x^{\prime}\left(2 \rho x^{\prime \prime}+3 x^{\prime}\right)+3\right)-\rho f^{\prime}}{\left(1+f x^{\prime 2}\right)^{2}}\right) .
\end{aligned}
$$

The next step is minimizing the entropy functional (3.9) in order to find the profile of the hypersurface which has been parametrized by $x(\rho)$. It is noted that $x(\rho)$ is supposed to be a smooth differentiable function with the condition $x(0)=\ell / 2$. To proceed, one may consider the entropy functional as a one-dimensional action in which the corresponding Lagrangian is independent of $x(\rho)$, which leads to a conservation law. In other words, let us write (3.9) as $S=\int d \rho \mathcal{L}$; thus the equation of motion becomes

$$
\frac{\partial}{\partial \rho}\left(\frac{\partial \mathcal{L}}{\partial x^{\prime \prime}}\right)-\left(\frac{\partial \mathcal{L}}{\partial x^{\prime}}\right)=C, \quad \text { with } \frac{\partial \mathcal{L}}{\partial x}=0,
$$

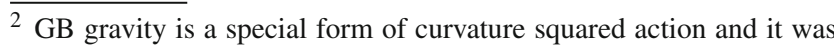
shown that for five-dimensional GB gravity, the proposal of computing HEE presented in [26] reduces to $[19,25]$ and the results are the same. Note that, taking into account the boundary term, only modifies the coefficient of the leading UV-divergent term.
} 
where $C$ is a constant which can be fixed by imposing the condition that at the turning point $\rho_{t}$ of the hypersurface in the bulk one has $x^{\prime}\left(\rho_{t}\right) \rightarrow \infty$. After minimizing the functional of (3.9) and using the condition of the hypersurface turning point, one gets the following conserved quantity along the radial profile:

$x^{\prime} \frac{1+f\left(x^{\prime 2}-2 \lambda\right)}{f\left(f^{-1}+x^{\prime 2}\right)^{3 / 2}}=\frac{\rho^{3}}{\rho_{t}^{3}}$.

In principle, the above equation allows us to find $x^{\prime}(\rho)$. In general, it is a difficult task to solve (3.11) to find a proper profile since it is a cubic equation for $x^{\prime}(\rho)$. However, in some special cases, the semianalytic solutions might be obtained. Up to the leading order of $\lambda$ and $\alpha$, and after making use of the following expression:

$\frac{\ell}{2}=\int_{0}^{\rho_{t}} x^{\prime}(\rho) d \rho$,

one obtains

$\ell=\frac{2 \sqrt{\pi}\left(1+\frac{3}{2} \lambda\right) \Gamma\left(\frac{2}{3}\right)}{\Gamma\left(\frac{1}{6}\right)} \rho_{t}+\frac{1}{12} \alpha^{2}\left(1-\frac{3}{2} \lambda\right) \rho_{t}^{3}$,

which can be inverted to find the turning point of the proposed hypersurface in the bulk as follows:

$$
\begin{aligned}
\rho_{t}= & \frac{\Gamma\left(\frac{1}{6}\right)}{2 \sqrt{\pi} \Gamma\left(\frac{2}{3}\right)} \ell-\frac{\Gamma\left(\frac{1}{6}\right)^{4}}{192 \pi^{2} \Gamma\left(\frac{2}{3}\right)^{4}} \ell^{3} \alpha^{2} \\
& +\left(\frac{-3 \Gamma\left(\frac{1}{6}\right)}{4 \sqrt{\pi} \Gamma\left(\frac{2}{3}\right)} \ell+\frac{5 \Gamma\left(\frac{1}{6}\right)^{4}}{128 \pi^{2} \Gamma\left(\frac{2}{3}\right)^{4}} \alpha^{2} \ell^{3}\right) \lambda \\
& +\mathcal{O}\left(\lambda^{2}, \alpha^{4}\right) .
\end{aligned}
$$

Plugging the results into (3.9), one gets the HEE as follows:

$S=\frac{H^{2} L^{3}}{4 G_{N}}\left(\frac{\mathfrak{a}}{\epsilon^{2}}+\mathfrak{b} \log \frac{\ell}{\epsilon}+\frac{\mathfrak{c}}{\ell^{2}}\right)+\mathcal{O}\left(\lambda^{2}, \alpha^{4}\right)$,

where $\epsilon$ stands for the UV scale which has been defined by the radial profile and

$$
\begin{aligned}
\mathfrak{a}=1 & -\frac{13}{2} \lambda, \\
\mathfrak{b}= & \frac{1}{4}\left(1-\frac{3}{2} \lambda\right) \alpha^{2}, \\
\mathfrak{c}=- & \frac{4 \pi^{3 / 2} \Gamma\left(\frac{2}{3}\right)^{3}}{\Gamma\left(\frac{1}{6}\right)^{3}}\left(1+\frac{9}{2} \lambda\right) \\
& +\frac{\ell^{2}}{4}\left(\left(1-\frac{3}{2} \lambda\right) \log \frac{\Gamma\left(\frac{1}{6}\right)}{2^{2 / 3} \sqrt{\pi} \Gamma\left(\frac{2}{3}\right)}-\frac{1}{3}+3 \lambda\right) \alpha^{2} .
\end{aligned}
$$

The leading divergent term in (3.15) is in fact the usual area law; on the other hand, the second term which is the universal logarithmic term is interesting. For a strip entangling region in $\mathrm{CFT}_{d>2}$, in principle, there is no such a universal term in the HEE. Nevertheless, due to the momentum relaxation parameter and GB coupling, up to $\mathcal{O}\left(\lambda^{2}, \alpha^{4}\right)$ one obtains a logarithmic universal term as follows: ${ }^{3}$

$S_{\text {univ. }}=\frac{H^{2} L^{3}}{16 G_{N}}\left(1-\frac{3}{2} \lambda\right) \alpha^{2} \log \frac{\ell}{\epsilon}$.

This term is physical and universal in the sense that it is not affected by a cutoff redefinition and can be used to introduce a modified ' $c$ '-type central charge in the corresponding dual quantum field theory. Using holographic entanglement entropy for the strip geometry, Myers and Singh [24] introduced a candidate for a $c$-function in arbitrary dimensions. In a $\mathrm{CFT}_{4}$ it goes as follows:

$c=\beta \frac{\ell^{3}}{H^{2}} \frac{\partial S(\ell)}{\partial \ell}$,

where $S(\ell)$ denotes the entanglement entropy for an interval of length $\ell$ and the precise value of $\beta$ has been identified by holographic calculations which is given by

$\beta=\frac{\Gamma\left(\frac{1}{6}\right)^{3}}{16 \pi^{3 / 2} \Gamma\left(\frac{2}{3}\right)^{3}}$.

In our setup by applying (3.18) and in the vicinity of $\lambda \bumpeq 0$, one obtains

$c=c(0)\left(1+\frac{9}{2} \lambda+\frac{\alpha^{2} \ell^{2}(2-3 \lambda) \Gamma\left(\frac{1}{6}\right)^{3}}{64 \pi^{3 / 2} \Gamma\left(\frac{2}{3}\right)^{3}}\right)$,

where $c(0)=\frac{\pi L^{3}}{8 G_{N}}$. Note that by turning off the momentum relaxation one gets the modified version of this function due to the GB term at the linear level, which was found in [24].

\subsubsection{Low-thermal excitation $\left(m \ell^{4} \ll 1\right)$}

In a special case of setting $\alpha=0$, we recover the fivedimensional GB AdS black brane solution with a Ricci-flat horizon which was found in [37]. On the other hand, for lowexcited state of CFT and near the UV boundary, (2.7) reduces to

$d s^{2}=\frac{\tilde{L}^{2}}{\rho^{2}}\left(-g(\rho) d \tau^{2}+\frac{1}{g(\rho)} d \rho^{2}+d X_{1}^{2}+d X_{2}^{2}+d X_{3}^{2}\right)$,

\footnotetext{
3 This was first observed in [15] and (3.17) is indeed its $\lambda$-correction.
} 
where $g(\rho)=1-m \rho^{4}+\mathcal{O}(m \lambda)$ and the modified AdS radius $\tilde{L}$ is defined by

$\tilde{L}^{2}=\frac{L^{2}}{f_{\infty}}, \quad$ where $\quad f_{\infty}=\frac{1-\sqrt{1-4 \lambda}}{2 \lambda}$.

An excited state due to such a deformation in CFT is called a thermal excitation. Thus, in particular, for the limit of $m \ell^{4} \ll$ 1 , the change of entropy can be obtained via the following relation:

$\Delta S=\left.\frac{H^{2} \tilde{L}^{2}}{4 G_{N}} \int d \rho\left(\frac{\delta \mathcal{S}}{\delta g}\right)\right|_{g=1} \Delta g$,

where $\mathcal{S}$, up to the leading order of GB coupling, is the integrand of entropy functional (3.9) given by

$$
\begin{aligned}
\mathcal{S}= & \frac{\sqrt{x^{\prime}(\rho)^{2}+g^{-1}(\rho)}}{\rho^{3}} \\
& \times\left(1-2 \lambda \frac{g(\rho)\left(g(\rho) x^{\prime}(\rho)\left(2 \rho x^{\prime \prime}(\rho)+3 x^{\prime}(\rho)\right)+3\right)-\rho g^{\prime}(\rho)}{\left(1+g(\rho) x^{\prime}(\rho)^{2}\right)^{2}}\right) .
\end{aligned}
$$

Therefore, one obtains

$\Delta S=S_{m \neq 0}-S_{0}=\frac{H^{2} \tilde{L}^{3}}{4 G_{N}} \frac{\left(1-6 \lambda f_{\infty}\right) \Gamma\left(\frac{1}{6}\right)^{2} \Gamma\left(\frac{1}{3}\right)}{40 \sqrt{\pi} \Gamma\left(\frac{2}{3}\right)^{2} \Gamma\left(\frac{5}{6}\right)} m \ell^{2}$,

where $S_{0}$ is the HEE for the vacuum case or pure AdS, namely $\alpha=\lambda=m=0$, and it is given by

$S_{0}=\frac{H^{2}}{4 G_{N}}\left(\frac{1}{\epsilon^{2}}-\frac{4}{\ell^{2}} \frac{\pi^{3 / 2} \Gamma\left(\frac{2}{3}\right)^{3}}{\Gamma\left(\frac{1}{6}\right)^{3}}\right)$.

The HEE (3.25) for low-thermal excitation due to the GB term will reproduce the result in [21].

\subsection{HEE of a sphere}

In this case, let us use the metric (2.7) with $\sum d x_{i}^{2}=d r^{2}+$ $r^{2} d \Omega_{2}^{2}$ in which $f(\rho)$ is given by (3.3). On the boundary, the entangling region is a sphere with radius $r<\ell$; therefore, the corresponding codimension two hypersurface in the bulk is realized by $t=0$ and $r=F(\rho)$. With this assumption, the induced metric becomes

$d s_{\text {ind }}^{2}=\frac{L^{2}}{\rho^{2}}\left[\left(F^{\prime 2}+f^{-1}\right) d \rho^{2}+r^{2} d \theta^{2}+r^{2} \sin ^{2} \theta d \phi^{2}\right]$.
Pursuing our previous example on strip entangling region, the corresponding entropy functional reads

$$
\begin{aligned}
S= & \frac{\pi L^{3}}{G_{N}} \int d \rho \frac{F^{2}}{\rho^{3}} \sqrt{\frac{1+f F^{\prime 2}}{f}}[1 \\
& +\frac{2 \lambda}{\left(1+f F^{\prime 2}\right)^{2}}\left(f^{2} F^{\prime}\left(4 \rho F^{\prime 2}-2 \rho F F^{\prime \prime}-3 F F^{\prime}\right)\right. \\
& +\rho f^{\prime}\left(F-\rho F^{\prime}\right)+\frac{\rho^{2}}{F} \\
& \left.\left.+f \rho\left(4 F^{\prime}-2 \rho F^{\prime \prime}+\frac{\rho F^{\prime 2}}{F}\right)-3 f F\right)\right] .
\end{aligned}
$$

By extremizing the obtained entropy functional and after making use of proper boundary conditions and for small parameters, the perturbative profile is found as follows ${ }^{4}$ :

$$
\begin{aligned}
F(\rho)= & \sqrt{\rho_{t}^{2}-\rho^{2}}\left[1-\frac{\lambda}{2}\right. \\
& +\alpha^{2} \frac{1}{24\left(\rho_{t}^{2}-\rho^{2}\right)^{3 / 2}}\left(12 \sqrt{\rho_{t}^{2}-\rho^{2}} \rho_{t}^{4} \log \left(\frac{\rho_{t}}{\rho}\right)\right. \\
& +\left(2 \rho_{t}^{2}-\rho^{2}\right)\left(\sqrt{\rho_{t}^{2}-\rho^{2}}\left(\rho^{2}+5 \rho_{t}^{2}\right)\right. \\
& \left.\left.\left.+6 \rho_{t}^{3} \log \left(\frac{\rho}{\sqrt{\rho_{t}^{2}-\rho^{2}}+\rho_{t}}\right)\right)\right)\right] .
\end{aligned}
$$

From the identity of $\ell=F(0)$ one can obtain the turning point in terms of the entangling region length. Thus, a semianalytic computation results in the following HEE for a sphere:

$$
\begin{aligned}
S= & \frac{\pi L^{3}}{2 G_{N}}\left[\frac{(2-13 \lambda) \ell^{2}}{2 \epsilon^{2}}-\frac{1}{4}(2-13 \lambda)-\frac{\alpha^{2} \ell^{2}}{3}\right. \\
& \left.+\frac{1}{4}\left(30 \lambda+\alpha^{2} \ell^{2}-4\right) \log \frac{2 \ell}{\epsilon}\right] .
\end{aligned}
$$

\subsection{HEE of a cylinder}

In the case of the cylinder, let us parameterize the metric (2.7) as

$$
\begin{aligned}
d s^{2}= & \frac{L^{2}}{\rho^{2}}\left(-f(\rho) d t^{2}+\frac{1}{f(\rho)} d \rho^{2}\right. \\
& \left.+d z^{2}+d r^{2}+r^{2} d \phi^{2}\right) .
\end{aligned}
$$

The entangling surface is a cylinder $r=\ell$ on the $t=0$ surface in this boundary geometry. We also introduce a regulator length $H$ for the $z$ direction which is along the cylinder length. By taking the profile as $r=F(\rho)$, the entropy functional becomes

\footnotetext{
${ }^{4}$ In the case of spherical and cylindrical entangling regions, we will consider the terms up to $\mathcal{O}\left(\lambda^{2}, \alpha^{4}, \lambda \alpha^{2}, m\right)$.
} 


$$
\begin{aligned}
S & =\frac{\pi H L^{3}}{2 G_{N}} \int d \rho \frac{F}{\rho^{3}} \sqrt{\frac{1+f F^{\prime 2}}{f}}\left(1-\lambda \mathcal{F}_{c y l .}\right), \\
\mathcal{F}_{c y l .} & =\frac{2 f^{2} F^{\prime}\left(F\left(2 \rho F^{\prime \prime}+3 F^{\prime}\right)-2 \rho\left(F^{\prime}\right)^{2}\right)+\rho f^{\prime}\left(\rho F^{\prime}-2 F\right)+2 f\left(\rho\left(\rho F^{\prime \prime}-2 F^{\prime}\right)+3 F\right)}{F\left(1+f F^{\prime 2}\right)^{2}} .
\end{aligned}
$$

Minimizing the above expression results in the following equation of motion:

$$
\begin{aligned}
& 2 f^{3} F^{\prime 2}\left(4 \lambda \rho F^{\prime 2}+F\left(-4 \lambda \rho F^{\prime \prime}-6 \lambda F^{\prime}+3 F^{\prime 3}\right)\right) \\
& \quad+2 f^{2}\left[F ^ { \prime } \left(\rho F^{\prime}\left(F^{\prime 2}+2 \lambda\right)\right.\right. \\
& \left.\left.\quad+6 F\left(F^{\prime 2}-\lambda\right)\right)-\rho F^{\prime \prime}\left(6 \lambda \rho F^{\prime}+F\left(F^{2}-2 \lambda\right)\right)\right] \\
& \left.\quad+\rho\left(f^{\prime} 2 \lambda \rho-F F^{\prime}\right)+2\right) \\
& \quad-f\left[4 \rho\left(F^{\prime 2}\left(\lambda \rho f^{\prime}-1\right)+\lambda\right)\right. \\
& \left.\quad+F\left(F^{\prime}\left(\rho f^{\prime}\left(F^{\prime 2}-6 \lambda\right)-6\right)+2 \rho F^{\prime \prime}\right)\right]=0
\end{aligned}
$$

which should be solved to obtain a proper profile, which is indeed a difficult task. However, to identify the universal contribution, the near boundary behavior of the minimal surface would be sufficient. Thus, the asymptotic solution of this equation can be considered as

$$
F(\rho)=c_{1}+c_{2} \rho+c_{3} \rho^{2}+\cdots,
$$

for which one finds

$$
c_{1}=\ell, \quad c_{2}=0, \quad c_{3}=-\frac{1}{4 \ell}+\frac{\lambda}{4 \ell}+\mathcal{O}\left(\lambda^{2}, \alpha^{4}\right),
$$

after making use of the boundary condition $F(0)=\ell$. By substituting the asymptotic form of the profile in (3.32), the universal part of HEE for the cylinder is finally obtained as follows:

$S_{\text {univ. }}=\frac{\pi H L^{3}}{2 G_{N}} \frac{-2+7 \lambda+2 \alpha^{2} \ell^{2}}{16 \ell} \log \frac{\ell}{\epsilon}$.

It is well known that the holographic computation of the Weyl anomaly could relate the gravity parameters $G_{N}$ and $\lambda$ to the central charges of dual CFTs [23]. In two dimensions, the central charge is related to the conformal anomaly via $\left\langle T_{\mu}^{\mu}\right\rangle=\frac{c}{24 \pi} R$, where $R$ is the Ricci scalar. In principle there are two trace anomaly coefficients in four-dimensional CFTs, namely $c$ - and $a$-functions

$$
\left\langle T_{\mu}^{\mu}\right\rangle \sim-a E_{4}+c W_{\alpha \beta \gamma \eta} W^{\alpha \beta \gamma \eta},
$$

where $E_{4}$ and $W_{\alpha \beta \gamma \eta}$ are, respectively, the Euler density and Weyl tensor. In this way it is argued that for a spherical entangling region the universal part of the HEE would be proportional to $a$ while for a cylindrical entangling region it relates to $c$-function [38]. However, for all AdS backgrounds, one obtains
$S_{\text {univ. }}=-\frac{\pi L^{3}}{2 G_{N}} \log \frac{2 \ell}{\epsilon} \equiv-4 a \log \frac{2 \ell}{\epsilon}$, for sphere,

$$
S_{\text {univ. }}=-\frac{\pi L^{3}}{16 G_{N}} \frac{H}{\ell} \log \frac{\ell}{\epsilon} \equiv-\frac{c}{2} \frac{H}{\ell} \log \frac{\ell}{\epsilon}, \quad \text { for cylinder, }
$$

which means for all theories dual to Einstein gravity that one gets the same central charges, namely $a=c=\frac{\pi L^{3}}{8 G_{N}}$. On the other hand, for higher-curvature gravity theories these coefficients get modified due to stringy corrections (see [39] for GB gravity). In our setup, a holographic computation shows that the coefficients of universal logarithmic terms have been modified as follows:

$$
\begin{aligned}
& c^{\circ}=c\left(1-\frac{7}{2} \lambda-\alpha^{2} \ell^{2}\right), \\
& { }^{\circ} a^{\circ}=a\left(1-\frac{15}{2} \lambda-\frac{1}{4} \alpha^{2} \ell^{2}\right) .
\end{aligned}
$$

This means that deforming the theory by adding highercurvature terms and some specific scalar fields to break the momentum conservation results in a change of the universal terms of the dual field theory. And the corresponding coefficients may be interpreted as the corrected (modified) central charges of the dual theory.

\section{Holographic $n$-partite Information and Wilson loop}

In addition to entanglement entropy, the $n$-partite information and also the Wilson loop are in fact useful quantities developed in the framework of gauge/gravity duality. In the case of two and three entangling regions, the $n$-partite information is equivalent to holographic mutual and tripartite information, respectively. These quantities indicate the amount of shared information or, more precisely, the correlation, between the entangling regions [40]. On the other hand, the Wilson loop is in fact another nonlocal operator which can be used as an important probe for studying phase structures of gauge theories. Investigating the effect of higher-order terms and momentum dissipation on these quantities is the main task of this section. 


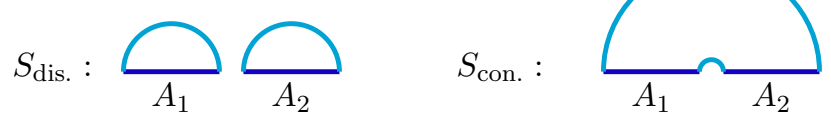

Fig. 1 Schematic representation of two different configurations for computing $S\left(A_{1} \cup A_{2}\right)$. In the case of a disconnected diagram $S\left(A_{1} \cup\right.$ $\left.A_{2}\right)=2 S(\ell)$, whereas for a connected diagram $S\left(A_{1} \cup A_{2}\right)=S(2 \ell+$ h) $+S(h)$

\subsection{Holographic mutual information}

For two separated systems, e.g., $A_{1}$ and $A_{2}$, the mutual information would be a proper measure for quantifying the amount of entanglement (or information) that these two systems can share. It is shown that, for two separated systems, the mutual information is a finite quantity and is given by [41]

$I\left(A_{1}, A_{2}\right)=S\left(A_{1}\right)+S\left(A_{2}\right)-S\left(A_{1} \cup A_{2}\right)$,

where $S\left(A_{1} \cup A_{2}\right)$ is the entanglement entropy for the union of two entangling regions. In quantum field theory, mutual information is used as a geometrical regularization of entanglement entropy. It is shown that holographic mutual information undergoes a first-order phase transition due to a discontinuity in its first derivative [42]. Holographically, this phase transition has in fact a simple geometrical explanation, e.g., for the union of two strips with the same length $\ell$ separated by distance $h$, there are two different configurations which are schematically shown in Fig. 1. It is worth mentioning that we have restricted ourselves to the case in which the entanglement entropy is an increasing function of the entangling region. Therefore, the mixed configurations have not been considered [43].

Depending on the value of $h / \ell$ the corresponding minimal configurations may change from one to another and this defines a critical ratio as $r_{c r i t} .=h / \ell$, in which

$S\left(A_{1} \cup A_{2}\right)=\left\{\begin{array}{cc}S_{\text {con. }} & 0<\frac{h}{\ell}<r_{\text {crit }} \\ S_{\text {dis. }} & r_{\text {crit. }} \leq \frac{h}{\ell} .\end{array}\right.$

Consequently, the holographic mutual information vanishes or takes a finite value which can be written as

$$
\begin{aligned}
I & \left(A_{1}, A_{2}\right) \\
& =\left\{\begin{array}{cc}
2 S(\ell)-S(h)-S(h+2 \ell), & 0<\frac{h}{\ell}<r_{\text {crit. }}, \\
0, & r_{\text {crit. }} \leq \frac{h}{\ell},
\end{array}\right.
\end{aligned}
$$

where in an $\mathrm{AdS}_{5}$ background one obtains $r_{\text {crit. }}=\sqrt{3}-1$. It is noted that in each case $S$ stands for HEE of the corresponding entangling region which is given by (3.15). Now the aim is to study the effect of $\alpha$ and $\lambda$ on the mutual information and its phase transition. In the presence of momentum dissipation and a GB term, let us write the mutual information as
$I\left(A_{1}, A_{2}\right)=I_{0}\left(A_{1}, A_{2}\right)+\Delta I\left(A_{1}, A_{2}\right)$,

where $I_{0}\left(A_{1}, A_{2}\right)$ stands for the mutual information when $\alpha=\lambda=0$, and after making use of the corresponding entanglement entropies for $\ell, h$ and $2 \ell+h$ regions from (3.15), it is obtained as follows:

$I_{0}=\frac{H^{2} L^{3}}{4 G_{N}} i_{0}$,
$i_{0}=\frac{4 \pi^{3 / 2} \Gamma\left(\frac{2}{3}\right)^{3}}{\Gamma\left(\frac{1}{6}\right)^{3}}\left(\frac{1}{(2 \ell+h)^{2}}+\frac{1}{h^{2}}-\frac{2}{\ell^{2}}\right)$.

On the other hand, the correction part becomes

$$
\begin{aligned}
\Delta I\left(A_{1}, A_{2}\right)= & \frac{H^{2} L^{3}}{4 G_{N}}\left(\left(1-\frac{3}{2} \lambda\right) \alpha^{2} i_{1}+\frac{9}{2} \lambda i_{0}\right) \\
& +\mathcal{O}\left(\lambda^{2}, \alpha^{4}\right),
\end{aligned}
$$

where

$i_{1}=\frac{1}{4} \log \frac{\ell^{2}}{h(2 \ell+h)}$.

As shown in Fig. 2, by turning on the momentum relaxation parameter, the mutual information between two regions decreases, whereas for a fixed momentum relaxation parameter, the mutual information linearly increases by the GB parameter.

Moreover, Fig. 3 shows the normalized curves of the phase transition as a function of GB and momentum relaxation parameters. One observes that, for a fixed momentum relaxation parameter, the phase transition of holographic mutual information takes place at larger distance by increasing the GB parameter (left plot in Fig. 3). The general behavior of the phase transition is decreasing by $\alpha$, though depending on the sign of the GB coupling $\lambda$, it behaves differently; for $\lambda>0$ the phase transition occurs in larger $h$ compared to the cases of $\lambda \leq 0$.

\subsection{Holographic tripartite information}

Besides mutual information, in a three-body system with topological order, tripartite information might be utilized as a quantity to characterize entanglement in the states of the system. It was first introduced in [44] as the topological entropy and defined by

$$
\begin{aligned}
I^{[3]}\left(A_{1}, A_{2}, A_{3}\right)= & S\left(A_{1}\right)+S\left(A_{2}\right)+S\left(A_{3}\right) \\
& -S\left(A_{1} \cup A_{2}\right)-S\left(A_{1} \cup A_{3}\right) \\
& -S\left(A_{2} \cup A_{3}\right)+S\left(A_{1} \cup A_{2} \cup A_{3}\right),
\end{aligned}
$$

where $S\left(A_{1} \cup A_{2} \cup A_{3}\right)$ is the entanglement entropy for the union of three subsystems. It is shown that the tripartite infor- 

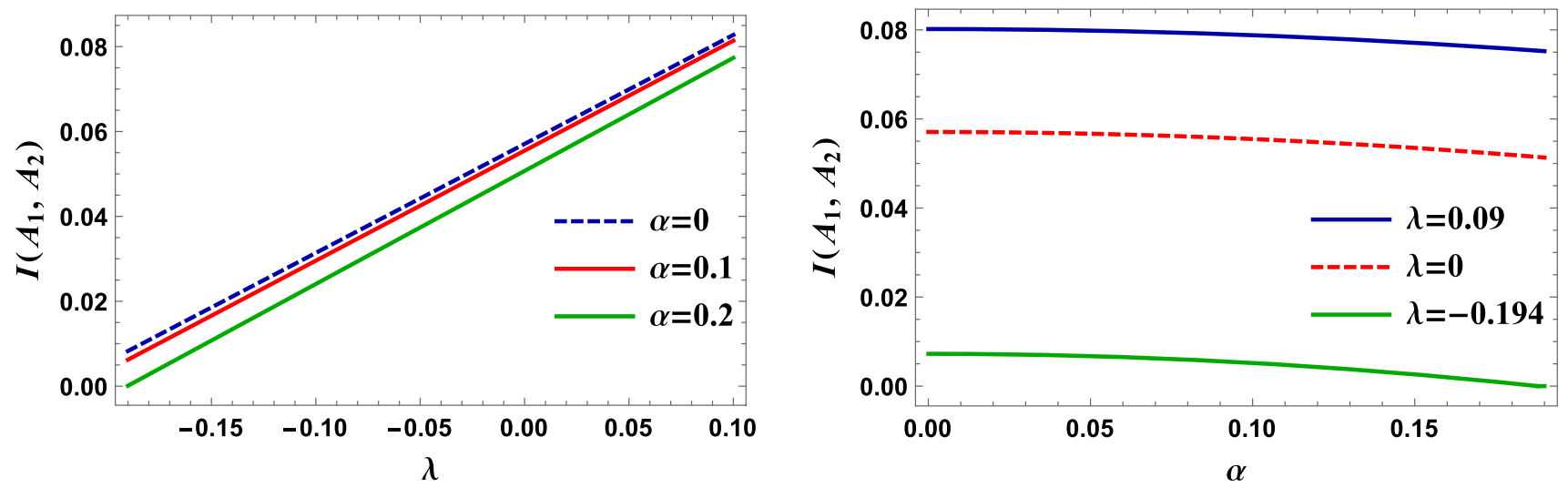

Fig. 2 Left plot: the behavior of mutual information as a function of GB parameter for different fixed values of $\alpha$. Right plot: the behavior of mutual information as a function of momentum relaxation parameter for different fixed values of $\lambda$. It is noted that in both of the figures we set $\ell=1$ and $h=0.7$
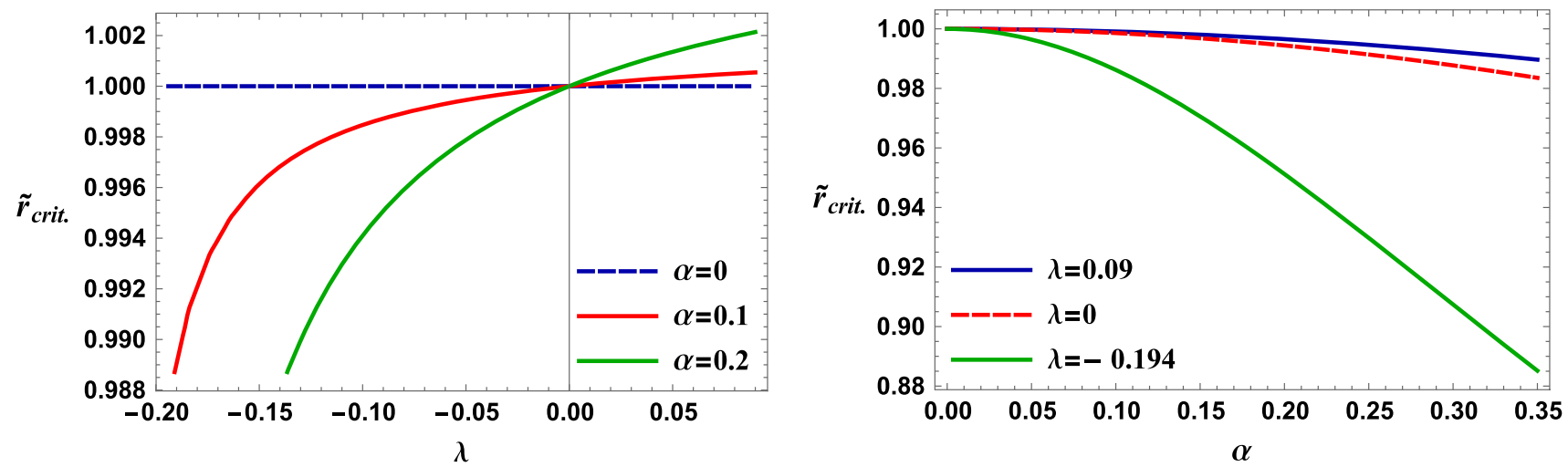

Fig. 3 Left plot: normalized transition curve $\tilde{r}_{\text {crit. }}=\frac{r_{\text {crit. }}}{r_{\text {crit. }}^{\lambda=0}}$ as a function of $\lambda$ for fixed values of $\alpha$. Right plot: normalized transition curve $\tilde{r}_{\text {crit. }}=\frac{r_{\text {crit. }}}{r_{\text {crit. }}^{\alpha=0}}$ as a function of $\alpha$ for different values of $\lambda$. It is noted that in both of the figures we set $\ell=1$

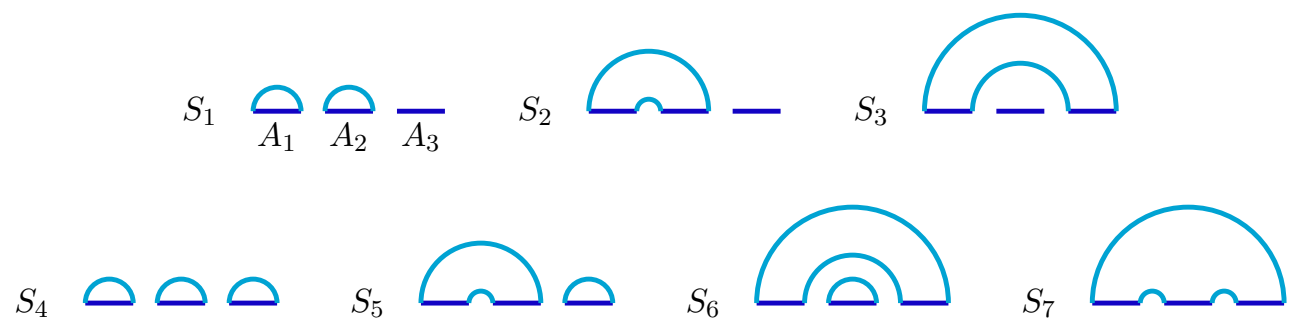

Fig. 4 Schematic representation of competing configurations in the computation of $S\left(A_{i} \cup A_{j}\right)$ and $S\left(A_{1} \cup A_{2} \cup A_{3}\right)$

mation is always finite even when the regions share boundaries. To compute the holographic tripartite information, the union terms of $S\left(A_{i} \cup A_{j}\right)$ and $S\left(A_{1} \cup A_{2} \cup A_{3}\right)$ deserve to be discussed further. For three strips, $A_{1}, A_{2}$ and $A_{3}$ of the same length $\ell$ separated by distance $h$, Fig. 4 shows schematically all possible diagrams for computing the union parts of the tripartite information. The rest of the configurations can be obtained by rearranging these.

Accordingly one obtains

$$
\begin{aligned}
& S\left(A_{i} \cup A_{j}\right)\left\{\begin{array}{l}
2 S(\ell) \equiv S_{1}, \\
S(2 \ell+h)+S(h) \equiv S_{2}, \\
S(3 \ell+2 h)+S(\ell+2 h) \equiv S_{3},
\end{array}\right. \\
& S\left(A_{1} \cup A_{2} \cup A_{3}\right)\left\{\begin{array}{l}
3 S(\ell) \equiv S_{4}, \\
S(3 \ell+2 h)+S(\ell+2 h)+S(\ell) \equiv S_{5}, \\
S(2 \ell+h)+S(\ell)+S(h) \equiv S_{6}, \\
S(3 \ell+2 h)+2 S(h) \equiv S_{7} .
\end{array}\right.
\end{aligned}
$$

Therefore, one can write the following expression for the tripartite information: 


$$
\begin{aligned}
I^{[3]}\left(A_{1}, A_{2}, A_{3}\right)= & 3 S(\ell)-2 \min \left\{S_{1}, S_{2}\right\} \\
& -\min \left\{S_{1}, S_{3}\right\}+\min \left\{S_{4}, S_{5}, S_{6}, S_{7}\right\} .
\end{aligned}
$$

As a special case when $\alpha=0$, Eq. (4.9) reduces to

$$
\begin{aligned}
& I^{[3]}\left(A_{1}, A_{2}, A_{3}\right) \\
& \quad=\left\{\begin{array}{cc}
S(\ell)-2 S(h+2 \ell)+S(2 h+3 \ell), & 0<\frac{h}{\ell}<r_{1}, \\
2 S(h)-3 S(\ell)+S(2 h+3 \ell), & r_{1} \leq \frac{h}{\ell}<r_{2}, \\
0, & r_{2} \leq \frac{h}{\ell},
\end{array}\right.
\end{aligned}
$$

where in the $\mathrm{AdS}_{5}$ background one finds two critical distances: $r_{1}=\sqrt{3}-1$ and $r_{2}=\frac{\sqrt{7}-1}{2}$, where the value of the tripartite information has been changed identically.

Similar to the mutual information, one can investigate that, in the presence of momentum relaxation parameter, the transition curves show a decreasing behavior with respect to $\alpha$ and $\lambda$, and for positive (negative) value of $\lambda$, the phase transition in tripartite information happens in a larger (smaller) ratio than the case of $\lambda=0$.

We end this subsection with a comment on a special property of tripartite information in holographic theories. The tripartite information can be written in terms of the mutual information as follows:

$$
\begin{aligned}
I^{[3]}\left(A_{1}, A_{2}, A_{3}\right)= & I\left(A_{1} \cup A_{2}\right) \\
& +I\left(A_{1} \cup A_{3}\right)-I\left(A_{1}, A_{2} \cup A_{3}\right) .
\end{aligned}
$$

For arbitrary states of systems, tripartite information has no definite sign, namely, depending on the underlying field theory, this quantity can be positive, negative or zero $[41,45,46]$. However, in strongly coupled CFTs with holographic duals it is argued that tripartite information is always negative $[47,48]$, and this property is related to the monogamy of the mutual information. ${ }^{5}$ In principle, it can be concluded that the holography leads to a constraint on this quantity and its sign might be employed in a variety of work (see for example $[49,50])$. In Fig. 5, we have plotted the tripartite information as a function of momentum relaxation parameter and GB coupling. One observes that it always remains negative. This behavior also holds when one changes the length of entangling regions for the given (fixed) values of momentum relaxation and GB coupling parameters.

\footnotetext{
${ }^{5}$ In the context of quantum information theory, the inequality of the form $F\left(A_{1}, A_{2}\right)+F\left(A_{1}, A_{3}\right) \leq F\left(A_{1}, A_{2} U A_{3}\right)$ is known as the monogamy relation. This feature of measurement is related to the security of quantum cryptography indicating that entangled correlations between $A_{1}$ and $A_{2}$ cannot be shared with a third system $A_{3}$ without spoiling the original entanglement [45].
}

\subsection{Wilson loop}

The Wilson loop has some properties similar to the entanglement entropy and it can be used to investigate the phase transitions in quantum systems. Namely this quantity characterizes phases of gauge theories in terms of the potential between electric charges. The expectation value of the Wilson loop, which is related to the effective potential between a quark and antiquark pair, can be approximated by the gauge/gravity correspondence as [51]

$$
\langle W\rangle \sim e^{-\frac{A(\Sigma)}{2 \pi \alpha^{\prime}}}
$$

where $\left(2 \pi \alpha^{\prime}\right)^{-1}$ is the string tension, $\Sigma$ is the string world sheet that extends in the bulk and $A(\Sigma)$ stands for the Nambu-Goto action for the string which by saddle point approximation reduces to the minimal surface of the classical string. Thus, for a rectangular Wilson loop of width $\ell$, the corresponding potential between the quark and antiquark is given by

$$
V=\frac{L^{2}}{\pi \alpha^{\prime}} \int_{\epsilon}^{\rho_{t}} d \rho \frac{\rho_{t}^{2}}{\rho^{2}} \sqrt{\frac{f(\rho)}{f(\rho) \rho_{t}^{4}-\rho^{4}}}
$$

where $f(\rho)$ is defined by (3.3). After doing the same computation as in the previous section, we find that

$$
\begin{aligned}
V= & V_{0}^{A d S}+\Delta V=\frac{L^{2}}{\alpha^{\prime}}\left[\frac{1}{\epsilon}-\frac{2 \Gamma\left(\frac{3}{4}\right)^{2}}{\Gamma\left(\frac{1}{4}\right)^{2}} \frac{1}{\ell}\right] \\
& +\frac{L^{2}}{\alpha^{\prime}}\left[\frac{\Gamma\left(\frac{3}{4}\right)^{2}}{\Gamma\left(\frac{1}{4}\right)^{2}} \frac{\lambda}{\ell}-\frac{\pi(3 \lambda+2) \alpha^{2} \ell}{192 \Gamma\left(\frac{3}{4}\right)^{4}}\right] .
\end{aligned}
$$

Therefore, after regularization and subtracting the effective potential of the AdS vacuum, the first-order correction of force between the quark and antiquark in this model is found as follows:

$$
\Delta F=-\frac{d}{d \ell} \Delta V=\frac{L^{2}}{\alpha^{\prime}}\left[\frac{\Gamma\left(\frac{3}{4}\right)^{2}}{\Gamma\left(\frac{1}{4}\right)^{2}} \frac{\lambda}{\ell^{2}}+\frac{\pi(3 \lambda+2) \alpha^{2}}{192 \Gamma\left(\frac{3}{4}\right)^{4}}\right]
$$

Noting that from the regularized part of AdS one receives an attractive force between these external particles, the correction due to the momentum relaxation is always repulsive and independent of $\ell$, which is in agreement with the results in [15]. However, the contribution of GB coupling is somehow nontrivial. The $\lambda$-correction part depends on separation $\ell$ and according to the sign of GB coupling could be either positive or negative which results in decreasing or increasing attractive force between quark and antiquark, respectively. 

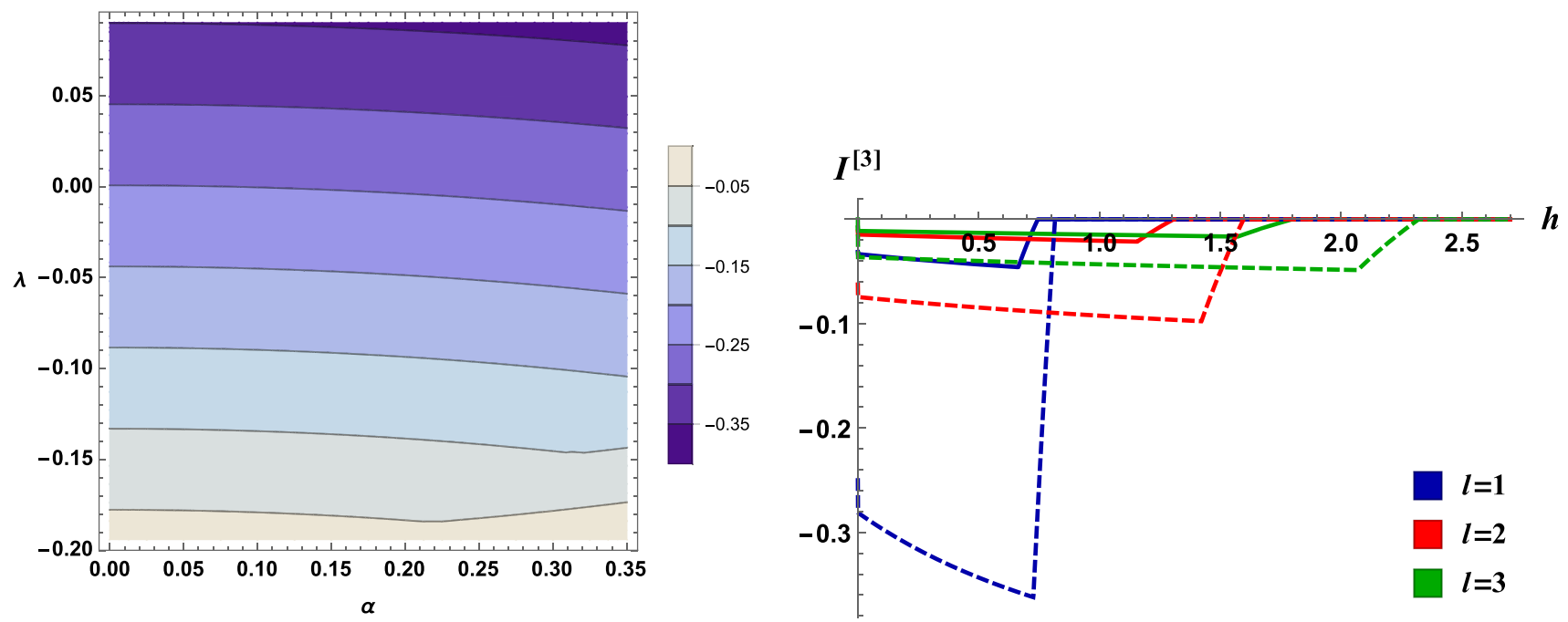

Fig. 5 Left plot: contour plot of tripartite information for $\ell=1$ and $h=0.7$. Right plot: tripartite information for $\alpha=0.3, \lambda=-0.194$ (solid curves) and $\lambda=0.09$ (dashed curves). In all ranges that we have considered $I^{[3]}<0$

\section{Conclusion}

In principle, in the holographic models, considering highercurvature terms in the gravity action is well motivated for several reasons; in particular, addressing different types of central charges could be an example. The Lovelock gravity is indeed the simplest set of higher-derivative terms in which various Euler densities appear as higher-derivative interactions in the gravity theory.

In this paper, we studied the effect of higher-order derivative terms on some nonlocal probes in the theories with momentum relaxation parameter. There are in fact two kinds of deformation in the states of dual field theory in this model: the higher-curvature terms, which could address the lowenergy quantum excitation corrections, and the deformation due to scalar fields, which are responsible for the momentum conservation breaking. We used holographic methods to obtain the corresponding changes due to these deformations in the coefficient of the universal part in the entanglement entropy. Higher-order gravity theories are interesting in the sense that they provide us with an effective description of quantum corrections and one may probe the finite coupling effects and the $a$ - and $c$-theorems via making such corrections to the Einstein gravity theory in the bulk space. We used fivedimensional Einstein GB gravity together with three spatially dependent massless scalar fields to obtain the corrections to universal and finite parts of HEE for strip, spherical and cylindrical entangling regions. For an interval of length $\ell$ on an infinite line, Myers and Singh introduced a candidate for the $c$-function in a $d$-dimensional CFT which is the coefficient of the finite term in entanglement entropy. This expression in $d=4$ is given by (3.18) and it can be considered as a function of the anomaly coefficients in the underlying CFT.
We showed that, in the presence of the momentum relaxation parameter and GB coupling, this expression has been modified as (3.20). Moreover, in computing the HEE for a strip, a universal logarithmic term appears due to the momentum relaxation parameter which has been modified by the GB coupling. This universal term vanishes at $\lambda \bumpeq 0.66$; however, noting that the GB coupling is constrained to a small range, i.e. $-0.194 \lesssim \lambda \leq 0.09$ [39,52], one gets a positive valued universal term due to both the momentum relaxation and the GB term in the present range. ${ }^{6}$

In the case of spherical entangling region, the coefficient of the universal term in HEE could potentially address the $a$-central charge of the corresponding dual conformal field theory whereas the $c$-central charge is related to the coefficient of the universal term in HEE for the cylindrical entangling region. For theories dual to Einstein gravity one obtains $a=c$; however, in the case of GB gravity one obtains unequal $a$ and $c$, this is indeed the main motivation of considering such a term in the gravity action. We obtained the modified coefficients of universal terms which can be interpreted as ' $c$ '-type central charge of dual field theory.

In the context of quantum information theory and also quantum many-body systems, for two disjointed systems, the mutual information is usually used as a measure of the quantum entanglement that these two systems can share; the mutual information can also be utilized as a useful probe to address certain phase transitions and critical behavior in these theories. For example, it is well known that mutual

\footnotetext{
${ }^{6}$ It is worth mentioning that considering the GB terms nonperturbatively leads to the violation of causality in any pure GaussBonnet gravity [53]. Moreover, we assume that momentum relaxation does not change the constraints on the GB coupling. We thank the referee for his/her useful comment on this point.
} 
information undergoes a transition beyond which it is identically zero; this kind of transition, which is called a disentangling transition, is in fact a universal qualitative feature for all classes of theories with holographic duals [54]. In this paper, we considered the effect of the GB term on such a phase transition in both the mutual and the tripartite information and it was shown that the behavior of such a phase transition is different, depending on the sign of GB coupling. For two strips with the same length separated by distance $h$, we showed that, for a fixed momentum relaxation parameter, the phase transition of holographic mutual information takes place at a larger distance by increasing the GB parameter. The general behavior of the phase transition is decreasing by $\alpha$, though for $\lambda>0$ the phase transition occurs in larger $h$ compared to the cases of $\lambda \leq 0$. For $\lambda>0$ this transition happens at a larger value than the case of $\lambda<0$. We also showed that the tripartite information has a negative value in our setup, which means that mutual information is monogamous.

Moreover, by considering the holographic Wilson loop, we found that the sign of $\lambda$ plays a crucial role in the computation of the effective potential and its corresponding force between point-like external objects. The result shows that both momentum dissipation and GB coupling parameters can lead to a correction of the potential and the corresponding force between quark and antiquark. Noting that from the regularized part of AdS one receives an attractive force between these external particles, the correction due to the momentum relaxation is always repulsive and independent of $\ell$, which is in agreement with the results in [15]. However, the contribution of GB coupling is somehow nontrivial. The $\lambda$-correction part depends on the separation $\ell$ and according to the sign of GB coupling could be either positive or negative, which results in a decreasing or an increasing attractive force between quark and antiquark, respectively.

Acknowledgements The author would like to thank Mohsen Alishahiha, M. Reza Mohammadi-Mozaffar and Ali Mollabashi for their helpful comments and discussions. MRT also wishes to acknowledge A. Akhvan, A. Faraji, A. Naseh, A. Shirzad, F. Omidi, F. Taghavi and M. Vahidinia for some their comments. We also thank the referees of this paper for their useful comments. This work has partially been supported by IAUCTB.

Open Access This article is distributed under the terms of the Creative Commons Attribution 4.0 International License (http://creativecomm ons.org/licenses/by/4.0/), which permits unrestricted use, distribution, and reproduction in any medium, provided you give appropriate credit to the original author(s) and the source, provide a link to the Creative Commons license, and indicate if changes were made.

Funded by SCOAP ${ }^{3}$.

\section{Appendix}

In this appendix, we write down some related computation of finding the entropy functional (3.9). In the present case there are two orthogonal normal vectors as follows:

$$
\begin{aligned}
& \Sigma_{1}: t=0 \quad n_{1}=\left\{\frac{\sqrt{f} L}{\rho}, 0,0,0,0\right\} \\
& \Sigma_{2}: x_{1}-x(\rho)=0 \quad n_{2}=\left\{0,-\frac{x^{\prime} L}{\rho \sqrt{f x^{\prime 2}+1}}, \frac{L}{\rho \sqrt{f x^{\prime 2}+1}}, 0,0\right\} .
\end{aligned}
$$

The corresponding extrinsic curvatures of the hypersurface are given by

$\mathcal{K}_{\mu \nu}^{(1)}=0, \quad \mathcal{K}_{\mu \nu}^{(2)}=L\left(\begin{array}{ccccc}0 & 0 & 0 & 0 & 0 \\ 0 & C_{1} f^{-1} & C_{1} x^{\prime} & 0 & 0 \\ 0 & C_{1} x^{\prime} & C_{1} f x^{\prime 2} & 0 & 0 \\ 0 & 0 & 0 & C_{2} & 0 \\ 0 & 0 & 0 & 0 & C_{2}\end{array}\right)$

where

$C_{1}=\frac{2\left(1+f x^{\prime 2}\right) f x^{\prime}-\rho\left(f^{\prime} x^{\prime}+2 f x^{\prime \prime}\right)}{2 \rho^{2}\left(1+f x^{\prime 2}\right)^{5 / 2}}$,

$C_{2}=\frac{f x^{\prime}}{\rho^{2} \sqrt{1+f x^{\prime 2}}}$.

Consequently, for a strip entangling region, the entanglement entropy of (2.4) for a general five-dimensional highercurvature gravity theory becomes

$S=\frac{H^{2} L^{3}}{4 G_{N}} \int d \rho \frac{\sqrt{x^{\prime 2}+f^{-1}}}{\rho^{3}}(1+\mathcal{A}+\mathcal{B})$,

where

$$
\begin{aligned}
\mathcal{A}= & \frac{-8(10 a+2 b+c) f+(32 a+7 b+4 c) \rho f^{\prime}-(4 a+b) \rho^{2} f^{\prime \prime}}{2 L^{2}} \\
& +\frac{\left[(3 b+4 c) \rho f^{\prime}-(b+4 c) \rho^{2} f^{\prime \prime}\right] f x^{\prime 2}}{2 L^{2}\left(1+f x^{\prime 2}\right)}, \\
\mathcal{B}= & -\frac{\rho^{4}\left[b\left(2 C_{2}+C_{1}\left(1+f x^{\prime 2}\right)\right)^{2}+4 c\left(2 C_{2}^{2}+C_{1}^{2}\left(1+f x^{\prime 2}\right)^{2}\right)\right]}{2 L^{2}} .
\end{aligned}
$$

By fixing the coupling constants of higher-order terms in (5.4), according to five-dimensional GB gravity, one obtains the entropy functional (3.9).

\section{References}

1. O. Aharony, S .S. Gubser, J .M. Maldacena, H. Ooguri, $\mathrm{Y}$. Oz, Large $\mathrm{N}$ field theories, string theory and gravity. Phys. Rep. 323, 183 (2000). https://doi.org/10.1016/ S0370-1573(99)00083-6. arXiv:hep-th/9905111

2. S.A. Hartnoll, Horizons, holography and condensed matter. arXiv: 1106.4324 [hep-th]

3. S .A. Hartnoll, Lectures on holographic methods for condensed matter physics. Class. Quant. Grav. 26, 224002 (2009). https://doi. org/10.1088/0264-9381/26/22/224002. arXiv:0903.3246 [hep-th] 
4. S.A. Hartnoll, J. Polchinski, E. Silverstein, D. Tong, Towards strange metallic holography. JHEP 1004, 120 (2010). https://doi. org/10.1007/JHEP04(2010)120. arXiv:0912.1061 [hep-th]

5. T. Faulkner, N. Iqbal, H. Liu, J. McGreevy, D. Vegh, From black holes to strange metals. arXiv:1003.1728 [hep-th]

6. S .A. Hartnoll, P.K. Kovtun, M. Muller, S. Sachdev, Theory of the Nernst effect near quantum phase transitions in condensed matter, and in dyonic black holes, Phys. Rev. B 76, 144502 (2007). https:// doi.org/10.1103/PhysRevB.76.144502. arXiv:0706.3215 [condmat.str-el]

7. A. Lucas, S. Sachdev, K. Schalm, Scale-invariant hyperscalingviolating holographic theories and the resistivity of strange metals with random-field disorder. Phys. Rev. D 89(6), 066018 (2014). https://doi.org/10.1103/PhysRevD.89.066018. arXiv:1401.7993 [hep-th]

8. R .A. Davison, Momentum relaxation in holographic massive gravity. Phys. Rev. D 88, 086003 (2013). https://doi.org/10.1103/ PhysRevD.88.086003. arXiv:1306.5792 [hep-th]

9. G .T. Horowitz, J .E. Santos, D. Tong, Optical conductivity with holographic lattices. JHEP 1207, 168 (2012). https://doi.org/10. 1007/JHEP07(2012)168. arXiv:1204.0519 [hep-th]

10. N. Bao, S. Harrison, S. Kachru, S. Sachdev, Vortex lattices and crystalline geometries. Phys. Rev. D 88(2), 026002 (2013). https:// doi.org/10.1103/PhysRevD.88.026002. arXiv:1303.4390 [hep-th]

11. M .R. Mohammadi Mozaffar, A. Mollabashi, Crystalline geometries from a fermionic vortex lattice. Phys. Rev. D 89(4), 046007 (2014). https://doi.org/10.1103/PhysRevD.89.046007. arXiv:1307.7397 [hep-th]

12. A. Lucas, Conductivity of a strange metal: from holography to memory functions. JHEP 1503, 071 (2015). https://doi.org/10. 1007/JHEP03(2015)071. arXiv:1501.05656 [hep-th]

13. T. Andrade, A simple model of momentum relaxation in Lifshitz holography. arXiv:1602.00556 [hep-th]

14. T. Andrade, B. Withers, A simple holographic model of momentum relaxation. JHEP 1405, 101 (2014). https://doi.org/10.1007/ JHEP05(2014)101. arXiv:1311.5157 [hep-th]

15. M Reza Mohammadi Mozaffar, A. Mollabashi, F. Omidi, Non-local probes in holographic theories with momentum relaxation. JHEP 1610, 135 (2016). https://doi.org/10.1007/JHEP10(2016)135. arXiv:1608.08781 [hep-th]

16. T. Nishioka, S. Ryu, T. Takayanagi, Holographic entanglement entropy: an overview. J. Phys. A 42, 504008 (2009). https://doi. org/10.1088/1751-8113/42/50/504008. arXiv:0905.0932 [hep-th]

17. V .E. Hubeny, M. Rangamani, T. Takayanagi, A covariant holographic entanglement entropy proposal. JHEP 0707, 062 (2007). https://doi.org/10.1088/1126-6708/2007/07/062. arXiv:0705.0016 [hep-th]

18. E. Abdalla, L .A. Correa-Borbonet, Aspects of higher order gravity and holography. Phys. Rev. D 65, 124011 (2002). https://doi.org/ 10.1103/PhysRevD.65.124011. arXiv:hep-th/0109129

19. J. de Boer, M. Kulaxizi, A. Parnachev, Holographic entanglement entropy in Lovelock gravities. JHEP 1107, 109 (2011). https://doi. org/10.1007/JHEP07(2011)109. arXiv:1101.5781 [hep-th]

20. N. Ogawa, T. Takayanagi, Higher Derivative corrections to holographic entanglement entropy for AdS solitons. JHEP 1110, 147 (2011). https://doi.org/10.1007/JHEP10(2011)147. arXiv:1107.4363 [hep-th]

21. W z Guo, S. He, J. Tao, Note on entanglement temperature for low thermal excited states in higher derivative gravity. JHEP 1308, 050 (2013). https://doi.org/10.1007/JHEP08(2013)050. arXiv:1305.2682 [hep-th]

22. M. Alishahiha, A .F. Astaneh, M .R. Mohammadi Mozaffar, Holographic entanglement entropy for 4D conformal gravity. JHEP 1402, 008 (2014). https://doi.org/10.1007/JHEP02(2014)008. arXiv:1311.4329 [hep-th]
23. M. Henningson, K. Skenderis, The holographic Weyl anomaly. JHEP 9807, 023 (1998). https://doi.org/10.1088/1126-6708/1998/ 07/023. arXiv:hep-th/9806087

24. R .C. Myers, A. Singh, Comments on holographic entanglement entropy and RG flows. JHEP 1204, 122 (2012). https://doi.org/10. 1007/JHEP04(2012)122. arXiv:1202.2068 [hep-th]

25. L .Y. Hung, R .C. Myers, M. Smolkin, On holographic entanglement entropy and higher curvature gravity. JHEP 1104, 025 (2011). https://doi.org/10.1007/JHEP04(2011)025. arXiv: 1101.5813 [hep-th]

26. D.V. Fursaev, A. Patrushev, S .N. Solodukhin, Distributional geometry of squashed cones. Phys. Rev. D 88(4), 044054 (2013). https:// doi.org/10.1103/PhysRevD.88.044054. arXiv:1306.4000 [hep-th]

27. X. Dong, Holographic entanglement entropy for general higher derivative gravity. JHEP 1401, 044 (2014). https://doi.org/10.1007/ JHEP01(2014)044. arXiv:1310.5713 [hep-th]

28. J. Camps, Generalized entropy and higher derivative gravity. JHEP 1403, 070 (2014). https://doi.org/10.1007/JHEP03(2014)070. arXiv: 1310.6659 [hep-th]

29. M R Mohammadi Mozaffar, A. Mollabashi, M.M. Sheikh-Jabbari, M .H. Vahidinia, Holographic entanglement entropy, field redefinition invariance and higher derivative gravity theories. Phys. Rev. D 94(4), 046002 (2016). https://doi.org/10.1103/PhysRevD. 94.046002. arXiv:1603.05713 [hep-th]

30. A. Ghodsi, M. Moghadassi, Holographic entanglement entropy from minimal surfaces with/without extrinsic curvature. JHEP 1602, 037 (2016). https://doi.org/10.1007/JHEP02(2016)037. arXiv:1508.02527 [hep-th]

31. P. Bueno, P .F. Ramirez, Higher-curvature corrections to holographic entanglement entropy in geometries with hyperscaling violation. JHEP 1412, 078 (2014). https://doi.org/10.1007/ JHEP12(2014)078. arXiv:1408.6380 [hep-th]

32. Y. Ling, P. Liu, J.P. Wu, Z. Zhou, Holographic metalinsulator transition in higher derivative gravity. Phys. Lett. B 766, 41 (2017). https://doi.org/10.1016/j.physletb.2016.12.051. arXiv:1606.07866 [hep-th]

33. R. Horodecki, P. Horodecki, M. Horodecki, K. Horodecki, Quantum entanglement. Rev. Mod. Phys. 81, 865 (2009). https://doi.org/ 10.1103/RevModPhys.81.865. arXiv:quant-ph/0702225

34. G. Vidal, J .I. Latorre, E. Rico, A. Kitaev, Entanglement in quantum critical phenomena. Phys. Rev. Lett. 90, 227902 (2003). https://doi. org/10.1103/PhysRevLett.90.227902. arXiv:quant-ph/0211074

35. M. Srednicki, Entropy and area. Phys. Rev. Lett. 71, 666 (1993). https://doi.org/10.1103/PhysRevLett.71.666. arXiv:hep-th/9303048

36. H. Casini, M. Huerta, Universal terms for the entanglement entropy in $2+1$ dimensions. Nucl. Phys. B 764, 183 (2007). https://doi.org/ 10.1016/j.nuclphysb.2006.12.012. arXiv:hep-th/0606256

37. R .G. Cai, Gauss-Bonnet black holes in AdS spaces. Phys. Rev. D 65, 084014 (2002). https://doi.org/10.1103/PhysRevD.65.084014. arXiv:hep-th/0109133

38. S .N. Solodukhin, Entanglement entropy, conformal invariance and extrinsic geometry. Phys. Lett. B 665, 305 (2008). https://doi.org/ 10.1016/j.physletb.2008.05.071. arXiv:0802.3117 [hep-th]

39. A. Buchel, J. Escobedo, R.C. Myers, M.F. Paulos, A. Sinha, M. Smolkin, Holographic GB gravity in arbitrary dimensions. JHEP 111, 2010 (1003). https://doi.org/10.1007/JHEP03(2010)111. arXiv:0911.4257 [hep-th]

40. A. Bernamonti, N. Copland, B. Craps, F. Galli, Holographic thermalization of mutual and tripartite information in 2d CFTs. PoS Corfu 2012, 120 (2013). arXiv:1212.0848 [hep-th]

41. H. Casini, M. Huerta, Remarks on the entanglement entropy for disconnected regions. JHEP 0903, 048 (2009). https://doi.org/10. 1088/1126-6708/2009/03/048. arXiv:0812.1773 [hep-th] 
42. M. Headrick, Entanglement Renyi entropies in holographic theories. Phys. Rev. D 82, 126010 (2010). https://doi.org/10.1103/ PhysRevD.82.126010. arXiv:1006.0047 [hep-th]

43. A. Allais, E. Tonni, Holographic evolution of the mutual information. JHEP 1201, 102 (2012). https://doi.org/10.1007/ JHEP01(2012)102. arXiv:1110.1607 [hep-th]

44. A. Kitaev, J. Preskill, Topological entanglement entropy. Phys. Rev. Lett. 96, 110404 (2006). https://doi.org/10.1103/PhysRevLett.96. 110404. arXiv:hep-th/0510092

45. P. Hayden, M. Headrick, A. Maloney, Holographic mutual information is monogamous. Phys. Rev. D 87(4), 046003 (2013). https:// doi.org/10.1103/PhysRevD.87.046003. arXiv:1107.2940 [hep-th]

46. M R Mohammadi Mozaffar, A. Mollabashi, F. Omidi, Holographic mutual information for singular surfaces. JHEP 1512, 082 (2015). https://doi.org/10.1007/JHEP12(2015)082. arXiv: 1511.00244 [hep-th]

47. M. Alishahiha, M R Mohammadi Mozaffar, M .R. Tanhayi, On the time evolution of holographic n-partite information. JHEP 165, 1509 (2015). https://doi.org/10.1007/JHEP09(2015)165. arXiv: 1406.7677 [hep-th]

48. S. Mirabi, M .R. Tanhayi, R. Vazirian, On the monogamy of holographic $n$-partite information. Phys. Rev. D 93(10), 104049 (2016). https://doi.org/10.1103/PhysRevD.93.104049. arXiv:1603.00184 [hep-th]
49. F. Pastawski, B. Yoshida, D. Harlow, J. Preskill, Holographic quantum error-correcting codes: toy models for the bulk/boundary correspondence. JHEP 1506, 149 (2015). https://doi.org/10.1007/ JHEP06(2015)149. arXiv:1503.06237 [hep-th]

50. A. Almheiri, X. Dong, D. Harlow, Bulk locality and quantum error correction in AdS/CFT. JHEP 1504, 163 (2015). https://doi.org/ 10.1007/JHEP04(2015)163. arXiv:1411.7041 [hep-th]

51. J .M. Maldacena, Wilson loops in large N field theories. Phys. Rev. Lett. 80, 4859 (1998). https://doi.org/10.1103/PhysRevLett. 80.4859. arXiv:hep-th/9803002

52. D .M. Hofman, J. Maldacena, Conformal collider physics: energy and charge correlations. JHEP 0805, 012 (2008). https://doi.org/ 10.1088/1126-6708/2008/05/012. arXiv:0803.1467 [hep-th]

53. X. O. Camanho, J. D. Edelstein, J. Maldacena, A. Zhiboedov, Causality constraints on corrections to the graviton threepoint coupling. JHEP 1602, 020 (2016). https://doi.org/10.1007/ JHEP02(2016)020. arXiv:1407.5597 [hep-th]

54. W. Fischler, A. Kundu, S. Kundu, Holographic mutual Information at finite temperature. Phys. Rev. D 87(12), 126012 (2013). https:// doi.org/10.1103/PhysRevD.87.126012. arXiv:1212.4764 [hep-th] 